\title{
Endometrial cancer presenting as a snowball in the pelvis
}

\author{
Rakesh Sinha $\cdot$ Meenakshi Sundaram • \\ Smita Lakhotia $\cdot$ Pratima Kadam
}

Received: 3 May 2010 / Accepted: 26 May 2010 / Published online: 11 June 2010

(C) Springer-Verlag 2010

Keywords Endometrial carcinoma

\section{Explanation image}

Endometrial cancer is the commonest gynaecologic malignancy in the west and the third commonest after cervical and ovarian cancer in India [1]. The standard treatment for endometrial cancer has been primary surgery. Locally advanced disease, comprising lymph node metastases, adjacent organ invasion, positive peritoneal cytology or serosal invasion is uncommon and accounts for only $7 \%$ of cases [2]. To categorise a patient as having serosal invasion, it is mandatory to have full-thickness myometrial invasion with macroscopic tumour on the uterine surface. We present a patient with serosal disease with spontaneous perforation of the uterine wall by tumour, which presented in a unique manner at laparoscopy.

A 66-year-old lady presented with post-menopausal vaginal bleeding on and off for 9 months. Imaging with transvaginal ultrasound and MRI revealed a uterine mass with endometrial thickness of $12 \mathrm{~mm}$. Under anaesthesia, a D\&C with frozen-section analysis revealed a grade II endometroid adenocarcinoma. At laparoscopy, a bulky

R. Sinha $\cdot$ M. Sundaram $\cdot$ S. Lakhotia $\cdot$ P. Kadam

Bombay Endoscopy Academy and Centre for Minimally Invasive Surgery (BEAMS Hospital),

Mumbai, India

M. Sundaram $(\square)$

674, 16th Cross Road, Behind Khar Gymkhana, Khar Pali,

Khar (W),

Mumbai 400 052, India

e-mail: drmeena25@yahoo.com

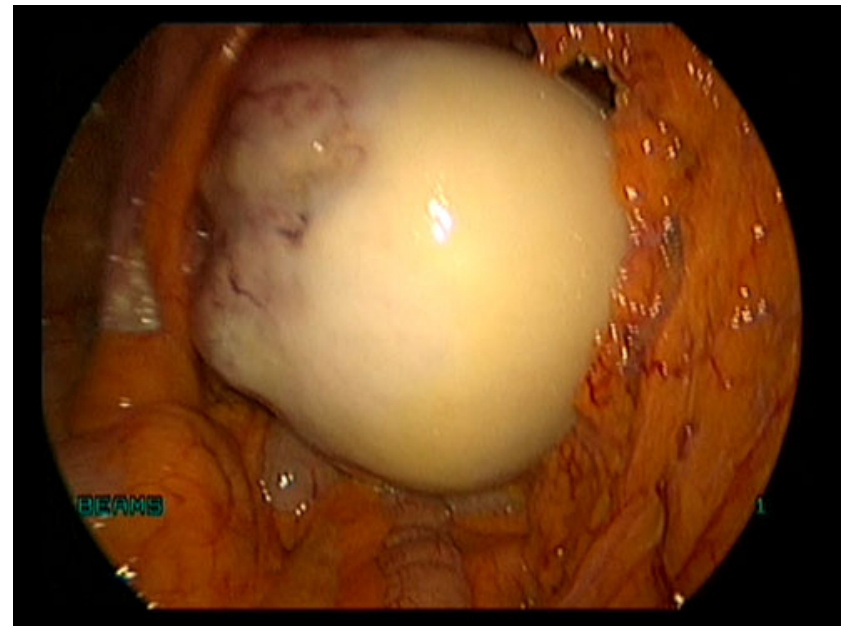

Fig. 1 Snowball in the pelvis

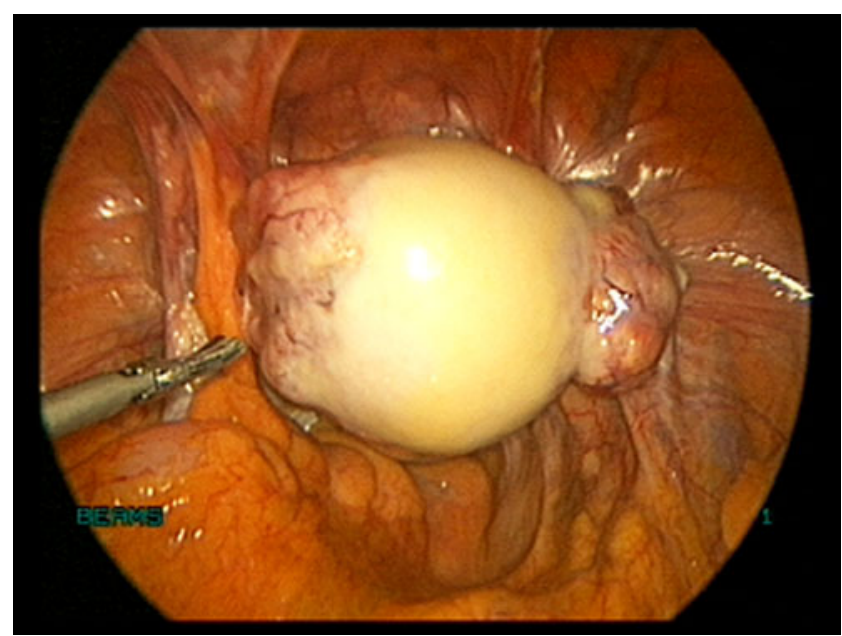

Fig. 2 Uterus covered by dense uniform whitish plaque 
uterus with tumour plaque on the uterine surface which had formed a dense white sheet was seen (Figs. 1 and 2). The right ovary was also enlarged. Standard surgical staging and pan hysterectomy was done. The permanent sections revealed a FIGO grade II endometroid adenocarcinoma with uterine serosal invasion and an ovarian deposit as well. There were no metastases in the pelvic nodes or outside the pelvis. The disease was staged as IIIA grade II. She made an uneventful recovery and is currently receiving chemoradiation. Review of literature did not reveal report of any similar case presenting like a snowball.
Declaration of interest The authors report no conflicts of interest. The authors alone are responsible for the content and writing of the paper.

\section{References}

1. Devi KU (2009) Current status of gynecological cancer care in India. J Gynecol Oncol 20(2):77-80

2. Ashman JB, Connell PP, Yamada D, Rotmensch J, Waggoner SE, Mundt AJ (2001) Outcome of endometrial carcinoma patients with involvement of the uterine serosa. Gynecol Oncol 82(2):338-343 